\title{
Large Squamous Papilloma of Buceal Mucosa
}

Naveena Kumar AN, Rajeswaran R, Harish Kumar H.

From the Department of Surgical Oncology, Jawaharlal Institute of Postgraduate Medical Education and Research (JIPMER), Pondicherry, India.

\section{Abstract:}

Oral squamous papilloma is a benign proliferation of the stratified squamous epithelium of mucosal surface, which appear as pedunculated or sessile, white or normal colored cauliflower-like projection. It seldom is large, with an average size less than $1.0 \mathrm{~cm}$. Many are only 3 to 4 millimeters. The most common sites are the palate- uvula area followed by tongue and lips. We report a rare case of large squamous papilloma arising from buccal mucosa measuring $7 \times 5 \times 2 \mathrm{~cm}$ size. Patient underwent surgical excision and required bipaddled sub mental flap to cover the defect in the oral cavity and cheek. The final histopathology reported as squamous papilloma.

Key words: Squamous papilloma, Buccal mucosa, Submental flap

\section{Introduction}

The squamous papillomas are benign exophytic masses of the oral cavity. It appears as pedunculated or sessile, white or normal colored cauliflower-like projections that arise from the mucosal surface. The common sites in the oral cavity are the palate, uvula, tongue and lips. The soft palate is the most common site and accounts for $20 \%$ of the lesions [1]. Most of the papillomas measure less than $1.0 \mathrm{~cm}$ [2]. Many are only 3 or 4 millimeters. The squamous papilloma arising from buccal mucosa larger than $7 \mathrm{~cm}$ have not been reported earlier in the literature. We report one such rare case of large squamous papilloma of oral cavity.

\section{Case Report}

A 65 year old lady presented with swelling in the right buccal mucosa since four years. Initially swelling was small in size and gradually progressed to present size [Fig.1]. There was no associated pain over the swelling. The patient complains of difficulty in speech and eating. The patient was non-alcoholic and non tobacco chewer. On examination there was a well defined verrucous growth in the right buccal mucosa extending to angle of mouth, measuring $7 \times 5 \mathrm{~cm}$. The upper and lower alveoli, retromolar trigone were free of the tumor. There was $2 \times 2$ $\mathrm{cm}$ well defined, firm, mobile, nontender lymph node palpable in the right sub mandibular region. Our clinical diagnosis was verrucous carcinoma. The incisional biopsy was taken from the lesion and reported to be squamous papilloma. The fine needle aspiration cytology from right level 1B lymph node reported to be reactive.

Patient underwent surgical excision and flap cover in view of large growth [Fig. 2]. The mucosal and cheek defect was closed with bi-paddled submental flap [Fig. 3, 4] which was based on submental vessels, branch of 


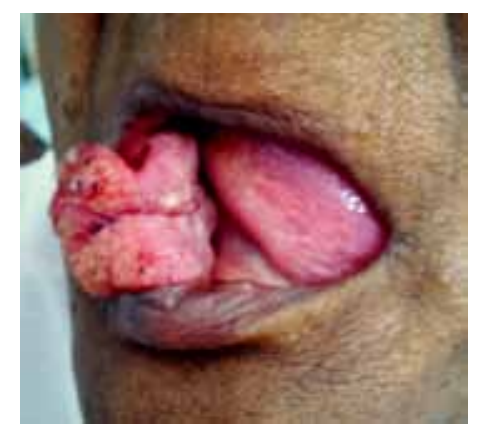

Fig.1: Squamous papilloma of buccal mucosa extending up to angle of mouth.

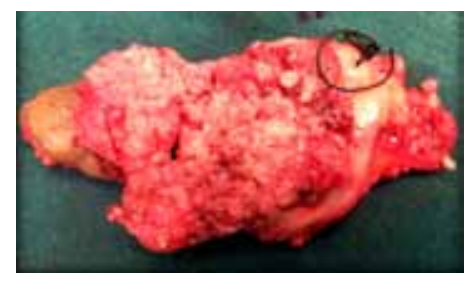

Fig.2: Resected large squamous papilloma of oral cavity.

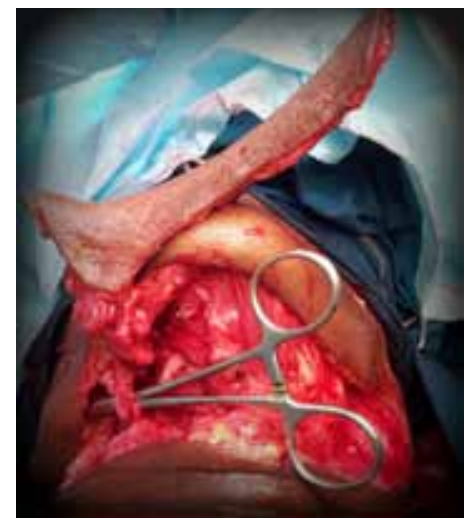

Fig.3: Pedicled submental flap with submental vessels.

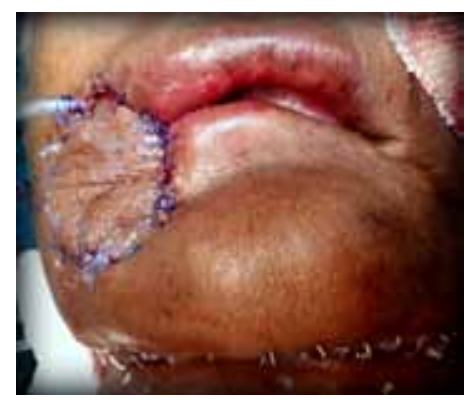

Fig.4: Bi-paddled submental flap covering mucosal and cheek defect. 
facial vessels. The final histopathology reported as squamous papilloma with dense lymphoid aggregates and giant cell reaction.

\section{Discussion}

Oral squamous papillomas are benign mucosal neoplasm. It is caused by viral infection of the epithelium, especially from human papilloma virus (HPV), most commonly HPV-6 and HPV-11 [3]. These subtypes are not associated with malignancy or pre-cancer. Several other subtypes of the human papilloma virus such as HPV-16, HPV-18, HPV-31, and HPV-45 could cause oral papilloma, might develop into oral cancer [4]. These double stranded DNA viruses are integrated with the DNA of host leading to papilloma.

The oral squamous papilloma is usually diagnosed in people between 20-50 years [5]. The common sites are the palate, uvula, tongue and lips. A single lesion is most common and appears as a soft, pedunculated mass with numerous finger-like projections. The projections may be long and pointy or short and rounded if keratin has built-up round the lesion. Less keratinized lesions are pink or red in colour and resemble a raspberry, while heavily keratinized lesions are white and look like the head of a cauliflower.

The oral squamous papilloma differs from other types of papilloma in a few important ways. While most of these growths are noncancerous in nature, a growth within the mouth does have a slightly higher likelihood of developing into a malignant mass. Oral papillomas also occur in greater frequency and are more likely to recur. As a result, they pose a potential respiratory risk if they overpopulate the throat. Papillomas found on the nasal or throat regions although sharing the same clinical features and histology as oral papillomas, differ in that usually more than one lesion is present, they proliferate continuously over time and often recur. The rare intraoral verruca vulgaris, condyloma acuminatum, and focal epithelial hyperplasia resemble papillomas and microscopic examination may be required to distinguish between them. Large papillomas may resemble early verrucous carcinoma [6].

Oral papillomas are painless and may be left untreated unless it is symptomatic. They appear not to change in size, spread to other parts of the oral cavity, or turn into malignant tumours. The keratinolytic agents containing lactic acid or a liquid nitrogen can be used for small benign cases. In case of large lesion conservative surgical excision may be performed. The recurrence is unlikely.

In conclusion, patients presenting with large exophytic growth of oral cavity, squamous papilloma should be considered as one of the differential diagnosis. Smaller, asymptomatic lesions should be managed conservatively while larger lesion requires surgical resection with/without soft tissue cover.

\section{Consent}

The informed consent is obtained from patient for publication of case report. 


\section{References}

1. Abbey LM, Page DG, Sawyer DR. The clinical and histopathological features of a series of 464 oral squamous cell papillomas. Oral Surg Oral Med Oral Pathol. 1980;49:419-428.

2. Prashant P. Jaju, Prashant V. Suvarna, Rajiv S. Desai.Squamous Papilloma: Case Report and Review of Literature. Int J Oral Sci. 2010;2(4):222-225.

3. StinaSyrjanen. Human papillomavirus infections and oral tumors. Med Microbiollmmunol. 2003;192:1 23128.

4. Thalassa E. Carneiro, Sandra A. Marinho, Flaviana D. Verli, Ana TM Mesquita, Nádia L. Lima, João L. Miranda. Oral squamous papilloma: clinical, histologic and immunohistochemical analyses. Journal of Oral Science 2009;51(3):367-372.

5. Anselmi AL, Premoli de Percoco G. Epidemiology study of 147 cases of oral Papilloma. Acta Odontol Venez. 1989;27(2-3):27-32. [Article in Spanish]

6. Koichi Mishima, Yoshiki Nariai, Seiji Obara, Yasuro Yoshimura. Human Papilloma virus in Oral Squamous Papilloma and Verrucous Carcinoma: Evaluation Using in Situ Polymerase Chain Reaction Detection. Asian J Oral Maxillofac Surg. 2002;1 4:95-99. 